\title{
Fas receptor signaling inhibits glycogen synthase kinase $3 \beta$ and induces cardiac hypertrophy following pressure overload
}

\author{
Cornel Badorff, ${ }^{1}$ Hartmut Ruetten, ${ }^{2}$ Sven Mueller, ${ }^{1}$ Meike Stahmer, ${ }^{1}$ Doris Gehring, ${ }^{2}$ \\ Frank Jung, ${ }^{1}$ Christian Ihling, ${ }^{3}$ Andreas M. Zeiher, ${ }^{1}$ and Stefanie Dimmeler ${ }^{1}$
}

\author{
${ }^{1}$ Molecular Cardiology, Department of Internal Medicine IV, University of Frankfurt, Frankfurt, Germany \\ ${ }^{2}$ Aventis Pharma, Frankfurt, Germany \\ ${ }^{3}$ Department of Pathology, University of Freiburg, Freiburg, Germany
}

Address for correspondence: Andreas M. Zeiher, Department of Internal Medicine IV, University of Frankfurt, Theodor Stern-Kai 7, 60590 Frankfurt, Germany.

Phone: 49-69-6301-5789; Fax: 49-69-6301-7113; E-mail: Zeiher@em.uni-frankfurt.de.

Cornel Badorff and Hartmut Ruetten contributed equally to this work.

Received for publication July 17, 2001, and accepted in revised form December 17, 2001.

Congestive heart failure is a leading cause of mortality in developed countries. Myocardial hypertrophy resulting from hypertension often precedes heart failure. Understanding the signaling underlying cardiac hypertrophy and failure is of major interest. Here, we identified Fas receptor activation, a classical death signal causing apoptosis via activation of the caspase cascade in many cell types, as a novel pathway mediating cardiomyocyte hypertrophy in vitro and in vivo. Fas activation by Fas ligand induced a hypertrophic response in cultured cardiomyocytes, which was dependent on the inactivation of glycogen synthase kinase $3 \beta$ (GSK3 $\beta$ ) by phosphorylation. In vivo, lpr (lymphoproliferative disease) mice lacking a functional Fas receptor demonstrated rapid-onset left ventricular dilatation and failure, absence of compensatory hypertrophy, and significantly increased mortality in response to pressure overload induction that was accompanied by a failure to inhibit GSK3 $\beta$ activity. In contrast, Fas ligand was dispensable for the development of pressure overload hypertrophy in vivo. In vitro, neonatal cardiomyocytes from $l p r$ mice showed a completely abrogated or significantly blunted hypertrophic response after stimulation with Fas ligand or angiotensin II, respectively. These findings indicate that Fas receptor signaling inhibits GSK3 $\beta$ activity in cardiomyocytes and is required for compensation of pressure overload in vivo.

J. Clin. Invest. 109:373-381 (2002). DOI:10.1172/JCI200213779.

\section{Introduction}

Cardiac hypertrophy occurs in response to the biomechanical stress of pressure overload, which is often caused by arterial hypertension. This adaptive growth is initially of a compensatory nature, but sustained hypertrophy later undergoes a transition to heart failure, a leading cause of mortality in developed countries $(1,2)$.

Apoptosis, leading to myocyte loss, is thought to contribute to experimental and clinical heart failure (3-5). Activation of the Fas/APO-1 receptor by Fas ligand represents a classical death signal causing apoptosis via activation of the caspase cascade in many cell types (6).

However, recent studies indicated that Fas signaling can have apoptosis-unrelated biological effects such as promotion of $\mathrm{T}$ cell proliferation (7). In cultured cardiomyocytes, Fas stimulation activates the prohypertrophic transcription factor AP-1 rather than inducing apoptosis (8). The mechanical stress induced by stretching enhances cardiomyocyte Fas expression of isolated papillary muscles (9). Upregulation of the myocardial Fas receptor was further described in both experimental volume-overload cardiac hypertrophy (8) and failing human hearts (10). Finally, in transgenic mice, myocardial overexpression of Fas ligand was shown to be sufficient to induce a hypertrophic phenotype (11). Collectively, these data suggest that Fas signaling appears to be involved in various forms of cardiac hypertrophy. However, the causative role of Fas receptor activation following pressure overload as well as the mechanism(s) of Fas-mediated hypertrophy in cardiomyocytes is unknown.

Therefore, we investigated the signaling pathways of Fas receptor activation in cultured cardiomyocytes and the cardiac phenotype of Fas signaling-defective lpr (lymphoproliferative disease) mice following pressure overload induction. Our results demonstrate that Fas activation-induced hypertrophy in cultured cardiomyocytes is dependent on phosphorylation and inactivation of glycogen synthase kinase $3 \beta$ (GSK3 $\beta$ ), a negative regulator of cardiomyocyte hypertrophy. Fas signaling is required for compensation of pressure overload in vivo, and its absence leads to failure to inhibit GSK3 $\beta$, rapid left ventricular dilatation, increased mortality, and absence of compensatory hypertrophy following this biomechanical stress. 

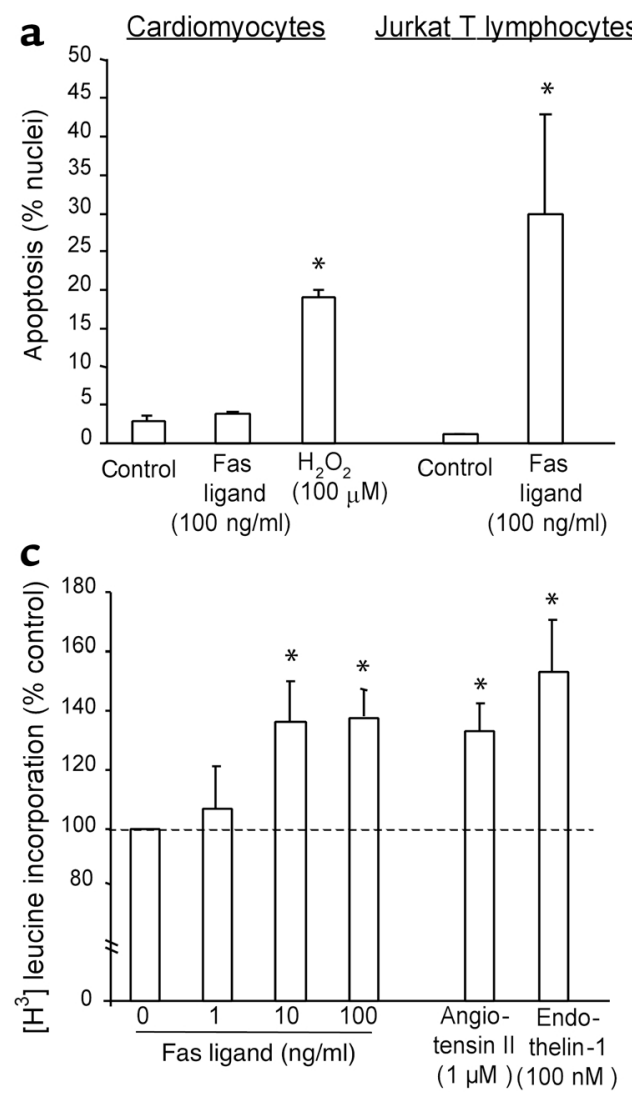

e FasL (10 ng/ml) Control $4 \mathrm{~h} \quad 12 \mathrm{~h} \quad 24 \mathrm{~h}$

ANF Northern blot

18S RNA
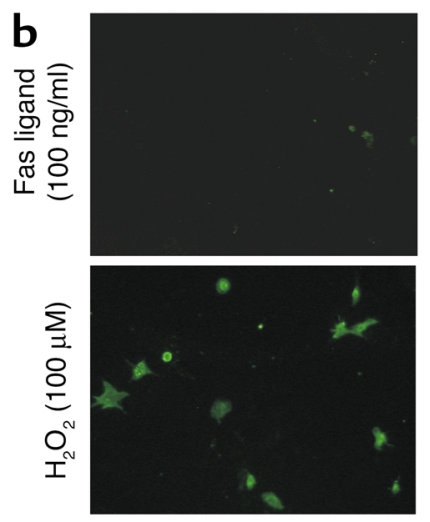

d
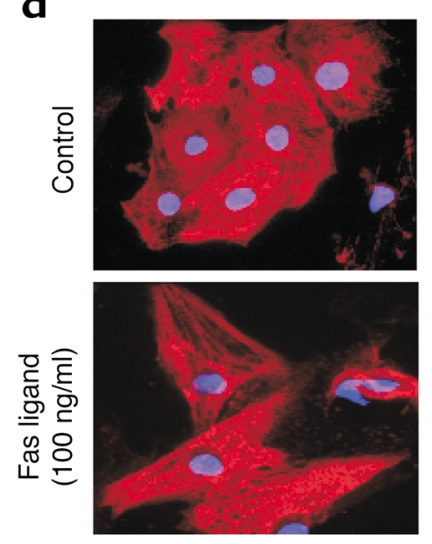

Figure 1

Fas ligand induces cardiomyocyte hypertrophy but not apoptosis. (a) Neonatal rat cardiomyocytes or Jurkat $\mathrm{T}$ lymphocytes were treated with Fas ligand or $\mathrm{H}_{2} \mathrm{O}_{2}$, and apoptosis was assessed by DAPI staining. Data are mean \pm SEM from four independent experiments. ${ }^{*} P<0.05$ versus control. (b) Annexin $V$ staining of rat cardiomyocytes following treatment with $\mathrm{Fas}$ ligand or $\mathrm{H}_{2} \mathrm{O}_{2}$. (c) $\left[{ }^{3} \mathrm{H}\right]$ leucine incorporation of rat cardiomyocytes following treatment with Fas ligand, angiotensin II, or endothelin-1. Data are mean \pm SEM from four independent experiments. ${ }^{*} P<0.05$ versus control. (d) Myosin light chain 2v immunofluorescence of rat cardiomyocytes following treatment with Fas ligand. (e) Increased ANF mRNA detected by Northern blotting following treatment with Fas ligand for the times indicated. Representative images of three independent experiments are shown in $\mathbf{c}-\mathbf{e}$.

\section{Methods}

Cell culture. Rat and mouse neonatal ventricular cardiomyocytes (12) and Jurkat T lymphocytes (13) were isolated and cultured as described. Following serum starvation for 18 hours, cells were stimulated with recombinant Fas ligand (1-100 ng/ml; Upstate Biotechnology Inc., Lake Placid, New York, USA), endothelin-1 (100 nM; Alexis Corp., San Diego, California, USA), angiotensin II ( $1 \mu \mathrm{M}$; Calbiochem-Novabiochem Corp., San Diego, California, USA), IGF-1 (40 ng/ml; Cell Concepts, Umkirch, Germany), or hydrogen peroxide (100 $\mu \mathrm{M})$. In some experiments, cells were preincubated for 60 minutes with $20 \mu \mathrm{M}$ Ly294002 or $200 \mathrm{nM}$ wortmannin (both from Calbiochem-Novabiochem Corp.). Apoptosis assays. In cultured cells, apoptosis was detected by DAPI and annexin V staining (13). In mouse hearts, DNA strand breaks were analyzed using TUNEL (14). Protein synthesis. One microcurie per milliliter $\left[{ }^{3} \mathrm{H}\right]$ leucine (Amersham Pharmacia Biotech, Piscataway, New Jersey, USA) was added 1 hour before stimulation with Fas ligand. Following lysis, proteins were precipitated using $5 \%$ trichloroacetic acid and $\left[{ }^{3} \mathrm{H}\right]$ leucine was measured by scintillation counting.

Immunoblotting. Cells or tissue were homogenized in lysis buffer, and $25 \mu \mathrm{g}$ was separated by SDS-PAGE, transferred to PVDF membranes, and immunoblotted as described (15). Primary antibodies were anti-phospho-Akt, anti-Akt, anti-phospho-GSK3 $\beta$ (all from Cell Signaling Technology, Beverly, Massachusetts, USA), anti-GSK3 $\beta$ (BD Transduction Laboratories, Los Angeles, California, USA), anti-phospho-JNK1, or anti-JNK1 (both from Santa Cruz Biotechnology Inc., Santa Cruz, California, USA). Bound antibodies were detected by enhanced chemiluminescence (Amersham Pharmacia Biotech).

Immunofluorescence staining and cell size measurement. Cardiomyocytes were stained with a rabbit anti-MLC2v antibody (a kind gift from Ju Chen, University of California-San Diego, San Diego, California, USA) followed by rhodamine-conjugated anti-rabbit IgG (Dianova, Hamburg, Germany). Nuclei were visualized by DAPI. Cells were imaged using an Axiovert 100M microscope equipped with an AxioCam (Carl Zeiss Jena GmbH, Jena, Germany). Maximum cell length and cell surface area were measured using Axiovision software (Carl Zeiss Jena $\mathrm{GmbH}$ ).

Atrial natriuretic factor Northern blotting, RT-PCR, and $R$ Nase protection assays. Total RNA was isolated (QIAGEN, Hilden, Germany) and Northern blot analysis was performed using a radioactively labeled, PCR-amplified full- 
length rat atrial natriuretic factor (ANF) cDNA probe. ANF mRNA was quantitated by real-time RT-PCR (Light Cycler; Roche Molecular Biochemicals, Indianapolis, Indiana, USA). RNase protection assays were from PharMingen (San Diego, California, USA).

Plasmid cloning, mutagenesis, transfection, and luciferase assay. The full-length human GSK3 $\beta$ cDNA was PCRamplified and cloned into pcDNA3.1-mycHis vector (Invitrogen Inc., Carlsbad, California, USA). Site-directed mutagenesis was performed with the Quick Change Mutagenesis Kit (Stratagene, La Jolla, California, USA). The entire 3.7-kb rat ANF promoter was PCR-amplified and cloned into pGL3-enhancer vector (Promega Corp., Madison, Wisconsin, USA). Plasmids were transfected into cardiomyocytes using Effectene (QIAGEN). Six hours after transfection, cells were serum-starved for 18 hours prior to stimulation with Fas ligand (50 $\mathrm{ng} / \mathrm{ml})$, endothelin-1 (10 $\mathrm{nM})$, or $\mathrm{LiCl}(10 \mathrm{mM})$ for 24 hours. Luciferase activity was measured using a commercial assay (Promega Corp.). Each data point is derived from an independent experiment and represents the mean of triplicate wells.

GSK3 $\beta$ and c-jun N-terminal kinase 1 immunocomplex kinase assay. Total GSK3 $\beta$ or c-jun $\mathrm{N}$-terminal kinase 1 (JNK1), respectively, was immunoprecipitated from myocardial homogenates using monoclonal antibodies, and the in vitro kinase assay was performed as described (16) with protein phosphatase inhibitor-2 (CalbiochemNovabiochem Corp.) as GSK3 $\beta$ substrate or GST-c-jun (Santa Cruz Biotechnology Inc.) as JNK1 substrate.

Animal experiments. C57BL $/ 6^{+/+}$, Fas-deficient C57BL/ $6^{l p r / / p r}$, and Fas ligand-deficient C57BL/6 ${ }^{\text {ld }} / g l d$ mice were obtained from The Jackson Laboratories (Bar Harbor, Maine, USA). Animal experiments were performed in accordance with the German animal protection law.

Pressure overload hypertrophy was induced by abdominal aortic constriction between the diaphragm and the renal arteries as described (17). Echocardiograms were performed in anesthetized mice, and, for

\section{Figure 2}

Fas ligand induces Akt and GSK3 $\beta$ phosphorylation in cardiomyocytes. (a) Rat cardiomyocytes were stimulated with IGF-1, endothelin-1, or Fas ligand for the times indicated. Lysates were immunoprobed for phospho-Akt or phospho-GSK3 $\beta$, followed by reprobes for total Akt or total GSK3 $\beta$, respectively. A representative blot from three independent experiments is shown. (b) Quantitative densitometric analyses of the phospho-Akt and phospho-GSK3 $\beta$ bands normalized for total Akt or GSK3. Data are mean \pm SEM from three independent experiments for each data point. ${ }^{*} P<0.05$ versus control. (c) Cardiomyocytes were pretreated with Ly294002 or wortmannin followed by stimulation with Fas ligand or IGF-1, immunoblotting with phospho-GSK3 $\beta$ antibody, and a reprobe for total GSK3 $\beta$. (d) Cardiomyocytes were cotransfected with an ANF promoter-driven luciferase reporter together with either empty vector (left) or a nonphosphorylatable GSK3 $\beta$ construct (GSK3 $\beta^{S 9 A}$ ) (right) followed by stimulation with Fas ligand. Data are mean \pm SEM from three to four independent experiments. ${ }^{*} P<0.05$ for stimulation versus control; ${ }^{\#} P<0.05$ for GSK3 $\beta^{\mathrm{S} 9 \mathrm{~A}}$ versus empty vector. FasL, Fas ligand. the assessment of pressure-volume relationships, the left ventricle was catheterized retrogradely using an impedance-pressure catheter (17).

Statistical analysis. Unpaired, two-tailed $t$ test was used for the comparison of two groups. For multiple groups, one-way ANOVA analysis (least significant difference test) was used. Mortality was analyzed by $\chi^{2}$ test.

\section{Results}

Fas receptor activation induces bypertrophy of cultured cardiomyocytes. As previously reported (6), recombinant Fas ligand caused apoptosis in Jurkat $\mathrm{T}$ lymphocytes after 6 hours with significantly increased nuclear fragmentation (Figure 1a). In contrast, Fas ligand did not result in increased apoptosis in neonatal rat ventricular cardiomyocytes, even after 18 hours, as measured by nuclear morphology (Figure 1a) and annexin $V$ staining (Figure 1b). Importantly, the functional integrity of the apoptosis execution program in neonatal cardiomyocytes was intact as evidenced by $\mathrm{H}_{2} \mathrm{O}_{2}$-induced

$\mathbf{a}$

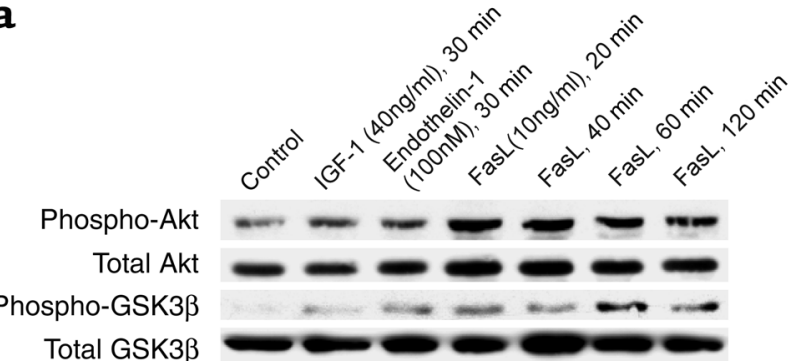

b
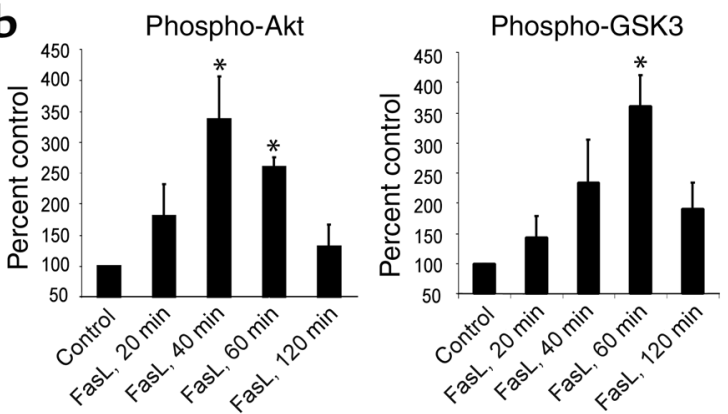

C

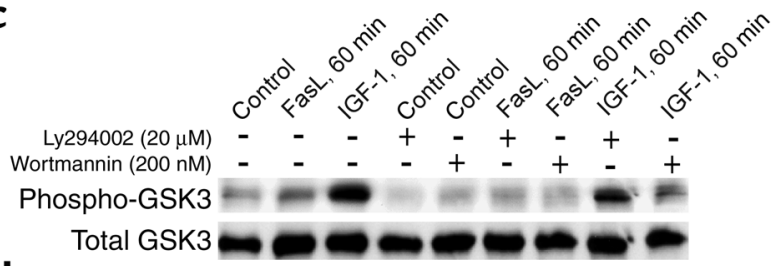

d

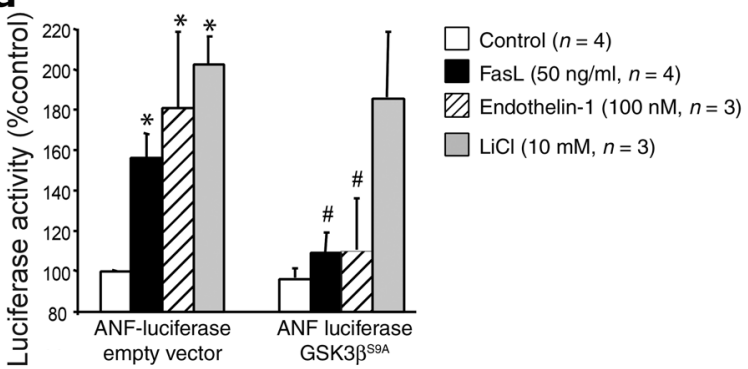



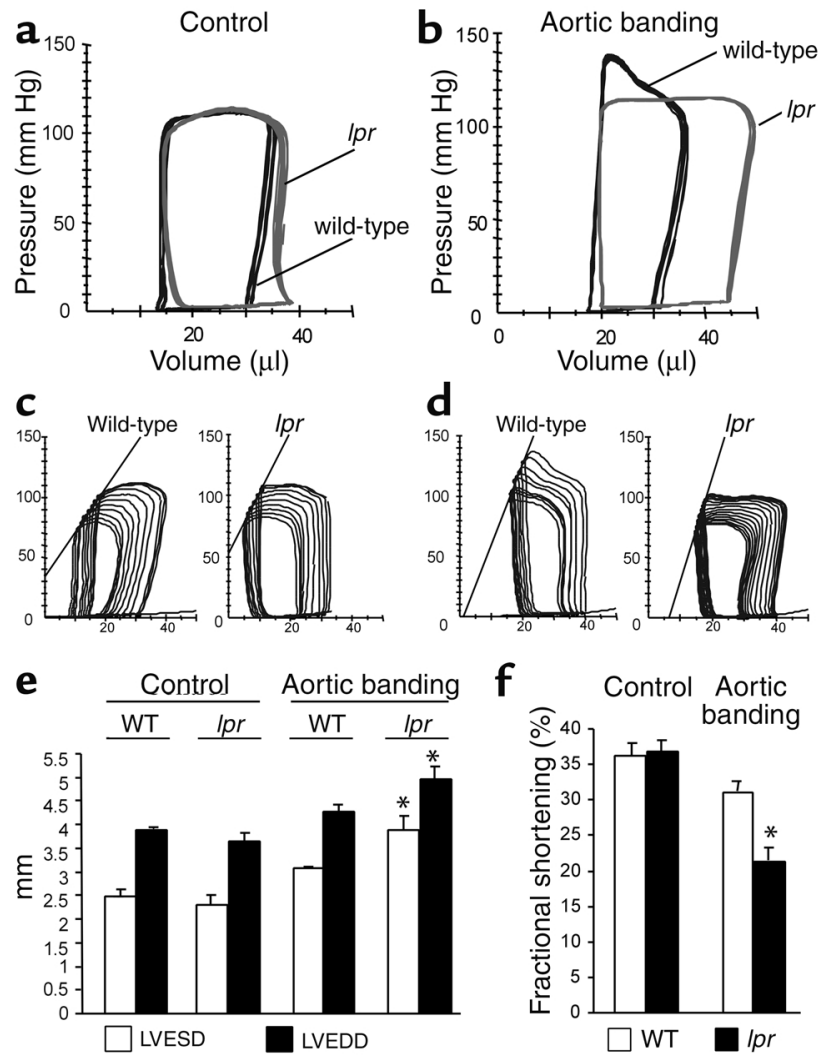

apoptosis (Figure 1, a and b). RNase protection assays confirmed that the Fas receptor and downstream apoptotic signaling molecules such as Fas-associated death domain and caspase- 8 were expressed in the neonatal cardiomyocytes used (data not shown).

Next, we investigated total protein synthesis, sarcomere organization, and gene induction of ANF as hallmarks of the hypertrophic response. Fas ligand dose-dependently $(1-100 \mathrm{ng} / \mathrm{ml})$ increased protein synthesis in neonatal cardiomyocytes after 24 hours. The increase in protein synthesis achieved by Fas ligand was comparable to the effects of well-established hypertrophic stimuli such as angiotensin II or endothelin-1 (Figure 1c). Fas ligand treatment profoundly increased cell size and sarcomeric organization in neonatal cardiomyocytes after 48 hours (Figure 1d). Furthermore, Fas ligand caused a time-dependent increase of ANF mRNA in cultured cardiomyocytes (Figure 1d). Thus, Fas signaling induces a hypertrophic response rather than apoptosis in neonatal cardiomyocytes.

Fas ligand-induced hypertrophy in cardiomyocytes is GSK3 $\beta$ dependent. Recent studies have shown that inactivation of GSK3 $\beta$ via phosphorylation, e.g., by the upstream kinase Akt, is both necessary and sufficient for cardiomyocyte hypertrophy in vitro $(18,19)$. As a result of GSK3 $\beta$ inactivation, the downstream effector nuclear factor of activated T cells (NF-AT) translocates to the nucleus to initiate hypertrophic gene expression (18).

In neonatal cardiomyocytes, Fas activation by Fas ligand $(10 \mathrm{ng} / \mathrm{ml})$ transiently increased Akt and GSK3 $\beta$

\section{Figure 3}

Heart failure of / pr mice in response to pressure overload. (a and $\mathbf{b}$ ) Representative, superimposed, steady-state pressure-volume loops of a wild-type (black) or Ipr mouse (gray) 2 days after sham-operation (a) or abdominal aortic banding (b). (c and $\mathbf{d}$ ) Representative pressure-volume loops during transient reduction of cardiac preload 2 days after sham-operation (c) or abdominal aortic banding (d). (e and $\mathbf{f}$ ) Echocardiographic measurements of surviving wild-type (WT) and Ipr mice 14 days after induction of pressure overload. The mean \pm SEM from six mice per group is shown. ${ }^{*} P<0.05$ for aortic banding versus control. LVEDD, left ventricular end-diastolic diameter; LVESD, left ventricular end-systolic diameter.

phosphorylation, as did endothelin-1 (100 nM) and IGF-1 $(40 \mathrm{ng} / \mathrm{ml})$, two established inducers of GSK3 $\beta$ phosphorylation $(18,19)$ (Figure 2a). Quantitative densitometric analysis revealed that Akt and GSK3 $\beta$ phosphorylation peaked 40 minutes and 60 minutes after addition of Fas ligand, respectively, consistent with the concept that Akt is upstream of GSK3 $\beta$ (Figure $2 \mathrm{~b}$ ). Downstream of GSK3 $\beta$, we observed a prominent nuclear translocation of NF-AT at 60 minutes after Fas receptor activation (data not shown). GSK3 $\beta$ phosphorylation induced by Fas ligand and IGF-1 was significantly inhibited by pretreatment (60 minutes) with Ly294002 or wortmannin, confirming a PI 3-kinasedependent (PI3K-dependent) pathway (Figure 2c).

To assess the causative role of GSK3 $\beta$ phosphorylation in Fas ligand-induced hypertrophy, neonatal cardiomyocytes were cotransfected with an ANF promoter-driven luciferase reporter and a nonphosphorylatable, dominant-active construct of GSK3 $\beta$ (GSK3 $\beta^{\mathrm{S} 9 \mathrm{~A}}$ ). In contrast to cotransfection with empty vector, the GSK3 $\beta^{\mathrm{S} 9 \mathrm{~A}}$ construct significantly suppressed Fas ligand-induced ANF promoter activation. Fas ligand- and endothelin1-induced ANF transcription was equally sensitive to the presence of GSK3 $\beta^{\mathrm{SPA}}$. In contrast, ANF induction by $\mathrm{LiCl}$ was unaffected by GSK3 $\beta^{\mathrm{S9A}}$ because $\mathrm{LiCl}$ inhibits GSK3 $\beta$ independent of its phosphorylation status (Figure $2 \mathrm{~d}$ ). Thus, Fas ligand-induced cardiomyocyte hypertrophy is GSK3 $\beta$-dependent.

Fas-deficient lpr mice develop congestive heart failure and enhanced mortality in response to pressure overload. Having demonstrated that Fas activation induces cardiomyocyte hypertrophy in vitro, we investigated the necessity of this pathway in Fas-deficient lpr mice after induction of pressure overload by abdominal aortic banding $(17,20)$. As control, wild-type strain $(\mathrm{C} 57 \mathrm{BL} / 6)$ sex- and age-matched mice were used.

Pressure-volume loops were recorded 2 days after sham-operation or abdominal aortic banding. Following sham-operation, wild-type and $l p r$ mice both had a normal cardiac phenotype with similar pressure-volume loops (Figure 3a) and hemodynamics (Table 1), ruling out a major physiological defect in lpr hearts at base line.

The trans-stenotic pressure gradients were comparable in wild-type $(39.1 \pm 5.3 \mathrm{mmHg})$ and $l p r$ mice $(37.6 \pm 6.4 \mathrm{mmHg})$, resulting in a significantly 

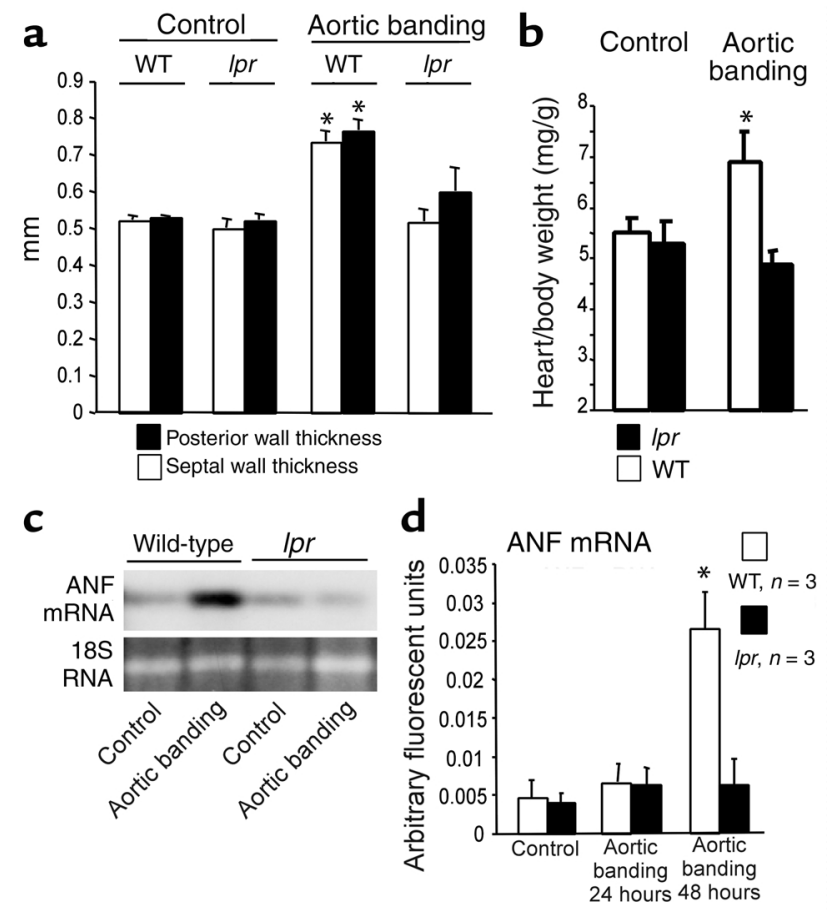

increased end-systolic pressure (ESP) and arterial elastance (Ea) in both groups (Table 1). Following 2 days of pressure overload, both wild-type and $l p r$ mice maintained their cardiac output (CO) with enhanced contractility during the isovolumetric phase $\left(\mathrm{dp} / \mathrm{dt}_{\max }\right)$ as well as the ejection phase, end-systolic pressure-volume relationship (Ees) and maximum chamber elasticity $\left(\mathrm{E}_{\max }\right)$, of the systole (Figure 3, $\mathrm{c}$ and d, and Table 1). However, already 2 days after induction of pressure overload, $l p r$ mice displayed significantly enlarged left ventricular end-diastolic volumes (Table 1), resulting in pressure-volume loops extended to the right (Figure 3, a and b) and a significantly increased preload recruitable stroke work (PRSW) (Table 1) as early indicators of impaired left ventricular hemodynamics.

Most importantly, 7 days after aortic banding, lethality rates were $55 \%$ (51/92 mice) in lpr mice compared with $17 \%$ (14/81 mice) in wild-type mice $(P<0.001)$.

Fourteen days after induction of pressure overload, surviving $l p r$ mice echocardiographically exhibited a significant increase of their left ventricular end-systolic and end-diastolic diameter (Figure 3e) as well as a significant decrease in fractional shortening (Figure 3f), demonstrating overt left ventricular failure.

\section{Table 1}

\section{Figure 4}

Cardiac hypertrophy in wild-type but not Ipr mice after pressure overload. (a) Echocardiographic septal (white bars) and posterior wall (black bars) thicknesses of wild-type and Ipr mice 14 days after induction of pressure overload. The mean \pm SEM from six mice per group is shown. ${ }^{*} P<0.05$ for aortic banding versus control. (b) Heart-tobody-weight ratios of wild-type (white bars) versus Ipr mice (black bars) 14 days after induction of pressure overload. The mean \pm SEM from six mice per group is shown. ${ }^{*} P<0.05$ for aortic banding versus control. ( $\mathbf{c}$ and $\mathbf{d}$ ) ANF mRNA expression 2 days after induction of pressure overload in wild-type and Ipr mice compared with shamoperated animals. (c) A representative ANF Northern blot (upper panel) is shown. 18S RNA (lower panel) demonstrates equal loading. (d) ANF mRNA group data derived from real-time RT-PCR of wild-type (white bars) and Ipr mice (black bars) at the times indicated following abdominal aortic banding. The mean \pm SEM from three mice per group is shown. ${ }^{*} P<0.05$ for aortic banding versus control.

Thus, Fas signaling is required for compensation of pressure-overload; its absence leads to rapid-onset left ventricular dilatation and failure as well as increased mortality.

Failure to develop pressure overload-compensating bypertrophy in mice lacking a functional Fas receptor. In order to morphologically assess the cardiac hypertrophic response to the induction of pressure overload, we determined left ventricular wall diameters by echocardiography. Following sham-operation, wild-type and $l p r$ mice had identical left ventricular wall thicknesses. Importantly, only wild-type but not $l p r$ mice responded to the induction of pressure overload with

Left ventricular mechanics as measured by pressure-volume loops of wild-type and Ipr mice 2 days after induction of pressure overload by aortic constriction

\begin{tabular}{|c|c|c|c|c|}
\hline \multirow[b]{2}{*}{ Parameter } & \multicolumn{2}{|c|}{ Wild-type } & \multicolumn{2}{|c|}{ Ipr } \\
\hline & $\begin{array}{l}\text { Sham } \\
(n=7)\end{array}$ & $\begin{array}{l}\text { Aortic banding } \\
\quad(n=7)\end{array}$ & $\begin{array}{l}\text { Sham } \\
(n=7)\end{array}$ & $\begin{array}{l}\text { Aortic banding } \\
\quad(n=7)\end{array}$ \\
\hline \multicolumn{5}{|l|}{ Hemodynamics } \\
\hline $\begin{array}{l}\operatorname{BW}(g) \\
\operatorname{HR}\left(\mathrm{min}^{-1}\right) \\
\operatorname{ESP}(\mathrm{mmHg}) \\
\operatorname{EDP}(\mathrm{mmHg}) \\
\operatorname{ESV}(\mu \mathrm{l}) \\
\operatorname{EDV}(\mu \mathrm{l}) \\
\operatorname{CO}(\mu \mathrm{l} / \mathrm{min})\end{array}$ & $\begin{aligned} 26.5 & \pm 0.7 \\
505 & \pm 23 \\
85.3 & \pm 5 \\
3.4 & \pm 0.6 \\
19.2 & \pm 5 \\
35.1 & \pm 5 \\
9862 & \pm 822\end{aligned}$ & $\begin{aligned} 26.0 & \pm 0.9 \\
562 & \pm 24 \\
126 & \pm 7^{A} \\
4.6 & \pm 0.9 \\
23.5 & \pm 4 \\
36.6 & \pm 5 \\
10544 & \pm 1022\end{aligned}$ & $\begin{aligned} 25.6 & \pm 0.8 \\
492 & \pm 33 \\
81.5 & \pm 8 \\
3.7 & \pm 0.7 \\
16.1 & \pm 3 \\
32.8 & \pm 4 \\
10160 & \pm 843\end{aligned}$ & $\begin{aligned} 26.11 & \pm 1.2 \\
532 & \pm 20 \\
116.8 & \pm 7^{\mathrm{A}} \\
5.6 & \pm 1 \\
22.2 & \pm 2^{\mathrm{A}} \\
41.8 & \pm 4^{\mathrm{A}} \\
14009 & \pm 1165\end{aligned}$ \\
\hline \multicolumn{5}{|l|}{ Systolic function } \\
\hline $\begin{array}{l}\mathrm{dp} / \mathrm{dt}_{\max }(\mathrm{mmHg} / \mathrm{s}) \\
\mathrm{Ea}(\mathrm{mmHg} / \mu \mathrm{l}) \\
\text { Ees }(\mathrm{mmHg} / \mu \mathrm{l}) \\
\text { PRSW } \\
\mathrm{E}_{\max }(\mathrm{mmHg} / \mu \mathrm{l})\end{array}$ & $\begin{aligned} 9412 & \pm 664 \\
3.1 & \pm 0.4 \\
3.0 & \pm 0.4 \\
65 & \pm 6 \\
6.37 & \pm 0.4\end{aligned}$ & $\begin{aligned} 14222 & \pm 1230^{\mathrm{A}} \\
7.1 & \pm 0.6^{\mathrm{A}} \\
4.4 & \pm 0.3^{\mathrm{A}} \\
62 & \pm 5 \\
13.6 & \pm 3.8^{\mathrm{A}}\end{aligned}$ & $\begin{aligned} 8550 & \pm 946 \\
3 & \pm 0.3 \\
3.4 & \pm 0.6 \\
55 & \pm 7 \\
4.3 & \pm 0.8\end{aligned}$ & $\begin{aligned} 12030 & \pm 1520^{\mathrm{A}} \\
4.6 & \pm 0.4^{\mathrm{A}, \mathrm{B}} \\
5.1 & \pm 1.3^{\mathrm{A}} \\
75 & \pm 8^{\mathrm{A}} \\
12.5 & \pm 2.6^{\mathrm{A}}\end{aligned}$ \\
\hline \multicolumn{5}{|l|}{ Diastolic function } \\
\hline $\begin{array}{l}\mathrm{dp} / \mathrm{dt}_{\min }(\mathrm{mmHg} / \mathrm{s}) \\
\tau(\mathrm{Glantz})(\mathrm{ms})\end{array}$ & $\begin{array}{c}9128 \pm 592 \\
6.1 \pm 0.2\end{array}$ & $\begin{aligned} 11457 & \pm 1230 \\
10.2 & \pm 1.8^{\mathrm{A}}\end{aligned}$ & $\begin{array}{c}8102 \pm 798 \\
7.1 \pm 0.2\end{array}$ & $\begin{array}{r}10020 \pm 724 \\
10.1 \pm 1.5^{\mathrm{A}}\end{array}$ \\
\hline
\end{tabular}

Shown are means \pm SEM. BW, body weight; HR, heart rate; ESP, end-systolic pressure; EDP, end-diastolic pressure; ESV, end-systolic volume; EDV, end-diastolic volume; $\mathrm{CO}$, cardiac output; $\mathrm{dp} / \mathrm{dt}_{\max }$, maximal rate of pressure development; $\mathrm{dp} / \mathrm{dt}_{\min }$, maximal rate of pressure decay; Ea, arterial elastance; Ees, end-systolic pressure-volume relationship; PRSW, preload recruitable stroke work; $E_{\max }$ maximum chamber elasticity; $\tau$, monoexponential time constant of relaxation. ${ }^{A} P<0.05$ aortic banding versus sham-operation; ${ }^{B} P<0.05$ Ipr versus wild-type after aortic banding. 
a

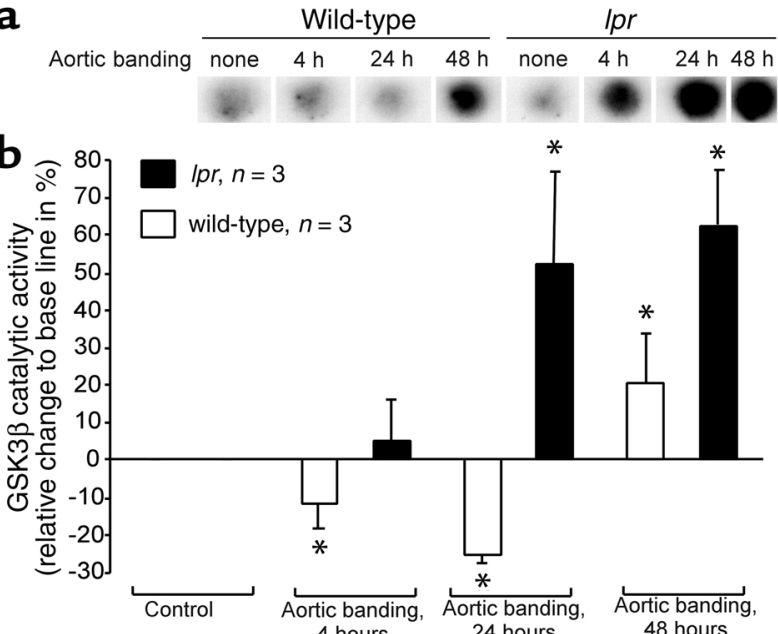

C

Aortic banding none $4 \mathrm{~h} 24 \mathrm{~h} 48 \mathrm{~h}$ none $4 \mathrm{~h} 24 \mathrm{~h} 48 \mathrm{~h}$ Phospho-Akt Total Akt

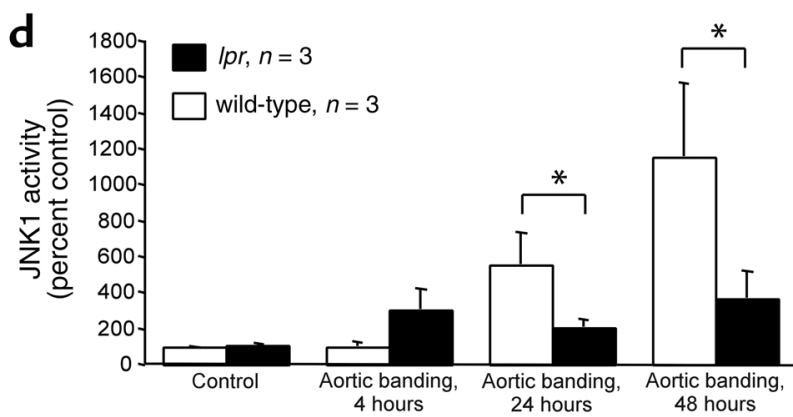

Figure 5

Lpr mice fail to inhibit GSK3 $\beta$ in response to pressure overload. (a) Representative GSK3 $\beta$ immunocomplex kinase assay dot blots of myocardial homogenates at the times indicated following aortic banding. (b) Group data derived from densitometric quantitation. Shown is the relative change (mean \pm SEM) in GSK3 $\beta$ activity compared with control in wild-type (white bars) and Ipr hearts (black bars); $n=3$ per group. ${ }^{*} P<0.05$ for aortic banding versus control. (c) Immunoblotting for phospho-Akt (top panel) followed by a reprobe for total Akt (bottom panel) of myocardial homogenates at the times indicated following induction of pressure overload. Shown is a representative blot from three animals in each group. (d) JNK1 activity in mouse heart homogenates following induction of pressure overload. Shown is the mean \pm SEM in wild-type (white bars) and $/ p r$ hearts (black bars); $n=3$ per group. ${ }^{*} P<0.05$ for wild-type versus $/ p r$.

significant increases of the septal as well as posterior wall thickness (Figure 4a). This hypertrophic response in the wild-type mice was confirmed by a significantly increased heart-to-body-weight ratio (Figure $4 \mathrm{~b}$ ). In contrast, wall thicknesses and the heart-to-bodyweight ratio remained unchanged in $l p r$ mice, indicating a failure to develop pressure overload-induced hypertrophy (Figure 4, a and b).

As a molecular marker of cardiomyocyte hypertrophy, we analyzed ANF mRNA induction at various times after aortic banding. Wild-type but not lpr mice responded to pressure overload with a robust induc- tion of ANF transcription (Figure 4c). Real-time RT-PCR revealed a significant fivefold increase of the ANF mRNA in wild-type but not lpr mice 2 days after induction of pressure overload (Figure 4d). These morphological and molecular data demonstrate that Fasdeficient lpr mice fail to develop adaptive cardiac hypertrophy in response to pressure overload.

Inbibition of GSK3 $\beta$ following pressure overload is abrogated in lpr mice. Next, we sought to identify the signaling pathway(s) defective in $l p r$ mice in response to pressure overload.

In order to determine whether altered phosphorylation and inactivation of GSK3 $\beta$ is involved in the failure of $l p r$ mice to develop compensatory left ventricular hypertrophy in response to the induction of pressure overload, GSK3 $\beta$ immunocomplex kinase assays of myocardial homogenates were performed at various time points following aortic banding. GSK3 $\beta$ activity was transiently but significantly reduced in wild-type mice 4 and 24 hours after induction of pressure overload. In contrast, $l p r$ mice exhibited an entirely different regulation of GSK3 $\beta$ activity, with a significant increase in GSK3 $\beta$ activity 24 hours after aortic constriction (Figure 5, a and b). Moreover, base-line GSK3 $\beta$ activity in the absence of pressure overload was lower in $l p r$ mice $(66.3 \% \pm 6.5 \%$ of wild-type mice, $P<0.05)$. Thus, in mice lacking a functional Fas receptor, pressure overload-induced inhibition of GSK3 $\beta$ activity is completely abrogated.

Because our cell culture studies as well as previous reports suggested that GSK3 $\beta$ inactivation involves phosphorylation by the kinase Akt $(18,19)$, we investigated the effects of pressure overload induction on Akt phosphorylation. Indeed, aortic banding induced a sustained phosphorylation of Akt up to 48 hours, which occurred in lpr mice to a lesser degree and more transiently (Figure 5c).

JNK1 activity and apoptosis in lpr mice following pressure overload. Since pressure overload activates multiple signaling pathways, and JNKs are known regulators of cardiac hypertrophy in vivo $(16,21)$, we determined the phosphorylation of JNK1, the predominant JNK isoform in the heart, as an index of kinase activity. Whereas wild-type mice showed a potent cardiac induction of JNK1 activity in response to pressure overload, the increase of JNK1 phosphorylation in lpr mouse hearts was significantly attenuated after 24 and 48 hours (Figure 5d). Consistent with these results, Fas receptor activation in cultured cardiomyocytes induced JNK1 activity about threefold after 40 minutes (data not shown).

Because Fas signaling causes apoptosis in various cell types, we assessed cardiomyocyte apoptosis by in situ TUNEL staining ( $n=6$ mice per group). In shamoperated animals, the percentage of $\mathrm{TUNEL}^{+}$cells was similar in $\operatorname{lpr}(0.39 \% \pm 0.2 \%)$ and wild-type mice $(0.4 \% \pm 0.1 \%)$. Although the number of apoptotic nuclei slightly increased 2 days after induction of pressure overload, there were no differences between wild-type $(0.76 \% \pm 0.4 \%)$ and $l p r$ mice $(1.1 \% \pm 1.5 \%)$. 
Given that $l p r$ mice develop a generalized lymphoproliferative disease, albeit at a considerably older age than the mice used for the present study, we immunohistochemically analyzed serial myocardial sections of lpr mice subjected to pressure overload for inflammatory infiltrates. Lpr mice did not reveal any myocardial inflammatory cells following aortic banding (data not shown). This suggests that the distinct cardiac response of $l p r$ mice to pressure overload is not due to a myocardial inflammatory process.

Fas ligand is dispensable for pressure overload-induced hypertrophy in vivo. Cardiac overexpression of Fas ligand, the prototypic binding partner of the Fas receptor, is sufficient to induce hypertrophy in transgenic mice (11). To test whether Fas ligand is necessary for pressure overload hypertrophy in a manner similar to the Fas receptor, we performed aortic constriction of homozygous gld (generalized lymphoproliferative disease) mice, which harbor a carboxyl-terminal Fas ligand point mutation rendering Fas ligand nonfunctional (20).

At base line, gld mice had ventricular dimensions and wall thicknesses similar to those of wild-type and $l p r$ mice (Figures 6 , $a$ and $b$ ). Following induction of pressure overload, gld mice maintained their ventricular dimensions without enlargement (Figure 6a), as well as their fractional shortening (data not shown). Gld mice responded to aortic banding with significant increases of the septal as well as posterior left ventricular wall (Figure 6b). This corresponded to a significantly increased left-ventricular-to-body-weight ratio 14 days after aortic banding $(4.02 \pm 0.31$ vs. $3.44 \pm 0.23 \mathrm{mg} / \mathrm{g}$, $n=8, P \leq 0.05)$. Most importantly, 7 days after aortic banding, the lethality rate of gld mice was $29 \%$ (4/14 mice, $P=$ not significant vs. wild-type, $P \leq 0.05$ vs. $l p r$ ). Thus, gld mice behave similarly to wild-type mice following the induction of pressure overload, indicating that Fas ligand is dispensable for the development of compensatory hypertrophy.

The Fas receptor mediates hypertrophy signaling beyond Fas ligand in vitro. The striking phenotypic disparity between $l p r$ and gld mice following aortic banding suggested that the Fas receptor may mediate hypertrophic signaling beyond Fas ligand. Therefore, we investigated this possibility in vitro using neonatal cardiomyocytes from wild-type and lpr mice.

Without stimulation, wild-type and $l p r$ cardiomyocytes had identical cell lengths and surface areas (Figure $6 \mathrm{~d}$ ). In conjunction with the results shown in Figure 1, wild-type cardiomyocytes showed a significant increase in both cell length and cell surface when treated with either Fas ligand or angiotensin II. Fas ligand induced a more elongated phenotype, whereas angiotensin II caused a greater increase in cell surface than in cell length. Expectedly, lpr cardiomyocytes were unable to respond to Fas ligand treatment. Importantly, the response of $l p r$ cardiomyocytes to angiotensin II was significantly blunted with no detectable increase in cell length and an increase of cell surface area, which was significantly lower than the one observed with wild-type cardiomyocytes (Figure 6d). These results indicate that the Fas receptor mediates hypertrophy signaling beyond Fas ligand in vitro, thus supporting our in vivo findings obtained with $l p r$ and gld mice.

\section{Discussion}

The results of the present study establish Fas receptor signaling as a novel pathway required for adaptive,
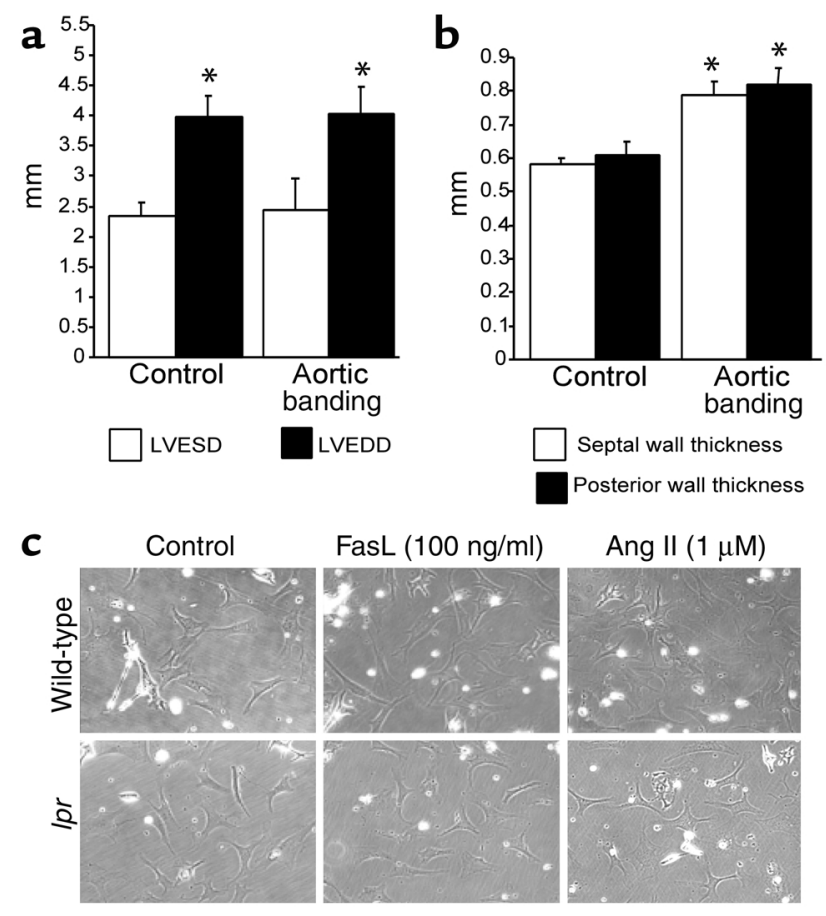

FasL $(100 \mathrm{ng} / \mathrm{ml}) \quad$ Ang II $(1 \mu \mathrm{M})$
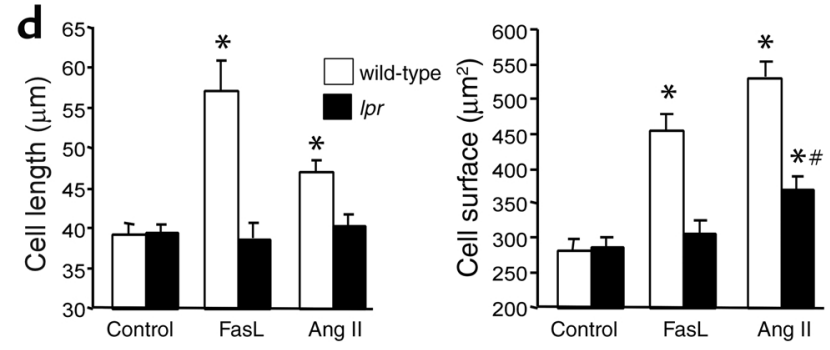

\section{Figure 6}

Fas ligand is dispensable for pressure overload hypertrophy in vivo, and Fas receptor mediates Fas ligand-independent signaling. (a) Echocardiographic left ventricular end-diastolic diameter (LVEDD) and end-systolic diameter (LVESD) of gld mice at base line (left, $n=4$ ) and 14 days after induction of pressure overload (right, $n=8$ ). The mean \pm SEM is shown. ${ }^{*} P \leq 0.05$ for aortic banding versus control. (b) Echocardiographic septal (white bars) and posterior wall (black bars) thicknesses of gld mice at base line (left, $n=4$ ) and 14 days after induction of pressure overload (right, $n=8$ ). The mean \pm SEM is shown. ${ }^{*} P \leq 0.05$ for aortic banding versus control. (c) Representative photomicrographs $(\times 400)$ of wild-type (top) or Fas-deficient I pr (bottom) mouse neonatal cardiomyocytes unstimulated (left) or stimulated with either Fas ligand (middle) or angiotensin II (Ang II; right) at the indicated concentrations for 48 hours. A typical experiment is presented. (d) Maximal cell length (left panel) and cell surface area (right panel) of wild-type (white bars) and Ipr cardiomyocytes (black bars). $n=10$ randomly chosen cells per group. ${ }^{*} P \leq 0.01$ for stimulation versus control; ${ }^{*} P \leq 0.01$ for wild-type versus $I p$. 
compensatory hypertrophy following the biomechanical stress of pressure overload. In vitro, Fas receptor activation caused cardiomyocyte hypertrophy depending on the inhibition of GSK3 $\beta$ activity by phosphorylation. In vivo, $l p r$ mice lacking a functional Fas receptor demonstrated abrogated GSK3 $\beta$ inhibition, rapid-onset left ventricular dilatation and failure, absence of compensatory hypertrophy, and significantly increased mortality following the induction of pressure overload. Results obtained with Fas ligand-defective gld mice and with $l p r$ cardiomyocytes in vitro indicate that the Fas receptor mediates signaling beyond its classical binding partner.

Although the Fas/Fas ligand system is a well-recognized and potent activator of apoptosis in many cell types (6), a growing body of evidence suggests cardiomyocyte-specific downstream signaling of the Fas receptor leading to survival and hypertrophy instead of apoptotic cell death. In cultured cardiomyocytes, Fas receptor activation was shown to activate the prohypertrophic transcription factor AP-1 (8). Transgenic mice overexpressing Fas ligand in the heart exhibit no enhanced cardiomyocyte apoptosis, but mild cardiac hypertrophy in a transgene dose-dependent manner (11). Our results with cultured rat and mouse neonatal cardiomyocytes indicate that Fas receptor activation with up to $100 \mathrm{ng} / \mathrm{ml}$ Fas ligand results in a specific elongated form of cardiomyocyte hypertrophy similar to the one observed with gp130 receptor stimulation and different from the phenotype evoked by angiotensin II (22). The concentrations of Fas ligand used did not cause cardiomyocyte apoptosis. However, much higher concentrations of Fas ligand (5000 $\mathrm{ng} / \mathrm{ml}$ ) can principally cause cardiomyocyte apoptosis, in particular when combined with doxorubicin (23). Consistent with a prominent role for Fas signaling to mediate hypertrophic responses in cardiomyocytes, the failure of $l p r$ mice lacking a functional Fas receptor to activate a compensatory hypertrophic response following induction of pressure overload indicates that Fas receptor signaling is required for at least this form of cardiac hypertrophy in vivo. The phenotype of rapidonset left ventricular dilatation and heart failure following induction of pressure overload accompanied by a substantially increased mortality observed in lpr mice is similar to the one of mice with cardiac-restricted gene targeting of gp130, a cell-surface receptor for cardiotrophin-1 (5). However, the underlying mechanisms appear to be different: In contrast to mice with cardiacrestricted loss of gp130, lpr mice do not develop increased cardiomyocyte apoptosis following pressure overload. In addition, lpr mice but not cardiac-restricted gp130-/- mice have lost the ability to activate a hypertrophic response on a molecular level (5).

Strikingly, Fas ligand-deficient gld mice behaved similarly to wild-type mice when challenged with pressure overload. First, this suggests that the phenotype observed in lpr mice did not occur secondarily due to autoimmunity disorders, since both $l p r$ and gld mice are prone to lymphoproliferative disorders (20). Second, the fact that Fas ligand is dispensable for pressure overload hypertrophy in vivo suggested that the Fas receptor may mediate signaling beyond Fas ligand. In support of these in vivo results, we found that neonatal cardiomyocytes from $l p r$ mice not only are unable to react to Fas ligand but also exhibit an impaired hypertrophy induction in response to angiotensin II. It is conceivable that the Fas receptor is involved in the transactivation of $G$ protein-coupled receptors, a phenomenon recently described for the bradykinin $\mathrm{B} 2$ receptor and VEGF receptor KDR/Flk-1 (24).

We now identified GSK3 $\beta$ as an important downstream cytoplasmic phosphorylation target of Fas receptor signaling. Phosphorylation and inactivation of GSK3 $\beta$, a negative regulator of cardiomyocyte hypertrophy, was recently identified as necessary and sufficient for hypertrophy induction by angiotensin II and endothelin-1 in vitro (18). Our in vitro studies demonstrate that increased ANF transcription induced by Fas activation as a hallmark of the hypertrophic response was dependent on GSK3 $\beta$ phosphorylation. Importantly, when compared with wild-type mice, $l p r$ mice displayed a differential GSK3 $\beta$ regulation with an increase rather than an inhibition of GSK3 $\beta$ activity following the induction of pressure overload. Therefore, inhibition of GSK3 $\beta$ activity appears to be also relevant in our in vivo model and may provide a molecular explanation for Fas activation-induced hypertrophy, which is defective in lpr mice.

GSK3 $\beta$ is an established target of the PI3K/Akt signaling pathway with Akt phosphorylating and thereby inactivating GSK3 $\beta$ (19). Fas receptor activation induced a profound and transient increase of Akt phosphorylation preceding the PI3K-dependent GSK3 $\beta$ phosphorylation. Downstream of GSK3 $\beta$, we observed a prominent nuclear translocation of NF-AT following Fas receptor activation. These data support previous studies in cardiomyocytes showing that GSK3 $\beta$ is downstream of Akt and that GSK3 $\beta$ phosphorylation occurs in a PI3K-dependent manner (19). However, the signaling pathway connecting the Fas receptor with the PI 3- and Akt-kinases remains to be elucidated.

Because cardiac hypertrophy is mediated by multiple signaling cascades, other signaling may also be altered in lpr mice in response to pressure overload. Indeed, we found that the potent JNK1 activation observed in wild-type mice after aortic banding was significantly attenuated in lpr mice. Given that JNKs are known regulators of cardiac hypertrophy in vivo $(16,21)$, JNK1 dysregulation may also contribute to the $l p r$ mouse heart phenotype observed. In this regard, it is notable that GSK3 $\beta$ is a negative regulator of the JNK target c-jun (25). Therefore, our JNK results are well compatible with an essential role for GSK3 $\beta$ in Fas-induced cardiomyocyte hypertrophy.

In summary, we identified Fas receptor signaling as a novel signal necessary for cardiac hypertrophy in response to the biomechanical stress of pressure over- 
load. In vitro, Fas activation-induced hypertrophy depends on increased GSK3 $\beta$ phosphorylation, and this mechanism may be causative in vivo as well.

\section{Acknowledgments}

We thank Isabel Lassotta-Klasen for expert technical assistance. This work was supported by the Deutsche Forschungsgemeinschaft (Ba1668/3-1, Ju241/2-1).

1. Hunter, J.J., and Chien, K.R. 1999. Signaling pathways for cardiac hypertrophy and failure. N. Engl. J. Med. 341:1276-1283.

2. Adams, K.F., Jr. 2001. New epidemiologic perspectives concerning mildto-moderate heart failure. Am. J. Med. 110(Suppl. 7A):6S-13S.

3. Narula, J., et al. 1996. Apoptosis in myocytes in end-stage heart failure. $N$ Engl. J. Med. 335:1182-1189.

4. MacLellan, W.R., and Schneider, M.D. 1997. Death by design: programmed cell death in cardiovascular biology and disease. Circ. Res. 81:137-144.

5. Hirota, H., et al. 1999. Loss of a gp130 cardiac muscle cell survival pathway is a critical event in the onset of heart failure during biomechanical stress. Cell. 97:189-198.

6. Nagata, S. 1997. Apoptosis by death factor. Cell. 88:355-365.

7. Kennedy, N.J., Kataoka, T., Tschopp, J., and Budd, R.C. 1999. Caspase activation is required for T cell proliferation. J. Exp. Med. 190:1891-1896.

8. Wollert, K.C., et al. 2000. The cardiac Fas (APO-1/CD95) Receptor/Fas ligand system: relation to diastolic wall stress in volume-overload hypertrophy in vivo and activation of the transcription factor AP-1 in cardiac myocytes. Circulation. 101:1172-1178.

9. Cheng, W., et al. 1995. Stretch-induced programmed myocyte cell death. J. Clin. Invest. 96:2247-2259.

10. Filippatos, G., et al. 1999. Expression of FAS adjacent to fibrotic foci in the failing human heart is not associated with increased apoptosis. Am.J. Physiol. 277:H445-H451.

11. Nelson, D.P., et al. 2000. Proinflammatory consequences of transgenic fas ligand expression in the heart. J. Clin. Invest. 105:1199-1208.

12. Badorff, C., et al. 1999. Enteroviral protease 2A cleaves dystrophin: evidence of cytoskeletal disruption in an acquired cardiomyopathy.
Nat. Med. 5:320-326.

13. Dimmeler, S., Haendeler, J., Nehls, M., and Zeiher, A.M. 1997. Suppression of apoptosis by nitric oxide via inhibition of ICE-like and CPP32-like proteases. J. Exp. Med. 185:601-608.

14. Weiland, U., et al. 2000. Inhibition of nitric oxide synthase potentiates ischemia/reperfusion-induced myocardial apoptosis via a caspase-3 dependent pathway. Cardiovasc. Res. 45:671-678.

15. Dimmeler, S., et al. 1999. Activation of nitric oxide synthase in endothelial cells via Akt-dependent phosphorylation. Nature. 399:601-605.

16. Choukroun, G., et al. 1999. Regulation of cardiac hypertrophy in vivo by the stress-activated protein kinases/c-Jun $\mathrm{NH}_{2}$-terminal kinases. J. Clin. Invest. 104:391-398.

17. Wettschurek, N., et al. 2001. Absence of pressure overload induced myocardial hypertrophy after conditional inactivation of $G \alpha_{q} / G \alpha_{11}$ in cardiomyocytes. Nat. Med. 7:1236-1240.

18. Haq, S., et al. 2000. Glycogen synthase kinase-3beta is a negative regulator of cardiomyocyte hypertrophy. J. Cell Biol. 151:117-130.

19. Cross, D.A., Alessi, D.R., Cohen, P., Andjelkovich, M., and Hemmings, B.A. 1995. Inhibition of glycogen synthase kinase- 3 by insulin mediated by protein kinase B. Nature. 378:785-789.

20. Takahashi, T., et al. 1994. Generalized lymphoproliferative disease in mice, caused by a point mutation in the Fas ligand. Cell. 76:969-976.

21. Esposito, $G$., et al. 2001. Cardiac overexpression of a $G_{q}$ inhibitor blocks induction of extracellular signal-regulated kinase and c-jun $\mathrm{NH}_{2}$-terminal kinase activity in in vivo pressure overload. Circulation. 103:1453-1458.

22. Wollert, K.C., et al. 1996. Cardiotrophin-1 activates a distinct form of cardiac muscle cell hypertrophy. Assembly of sarcomeric units in series via gp130/leukemia inhibitory factor receptor-dependent pathways. J. Biol. Chem. 271:9533-9545.

23. Yamaoka, M., et al. 2000. Apoptosis in rat cardiac myocytes induced with Fas ligand: priming for Fas-mediated apoptosis with doxorubicin. J. Mol. Cell. Cardiol. 32:881-889.

24. Thuringer, D., Maulon, L., and Frelin, C. 2002. Rapid transactivation of the VEGF receptor KDR/Flk-1 by the bradykinin B2 receptor contributes to eNOS activation in cardiac capillary endothelial cells. J. Biol. Chem. 277:2028-2032. DOI:10.1074/jbc.M109493200.

25. Troussard, A.A., Tan, C., Yoganathan, N., and Dedhar, S. 1999. Cell-extracellular matrix interactions stimulate the AP-1 transcription factor in an integrin-linked kinase- and glycogen synthase kinase 3-dependent manner. Mol. Cell. Biol. 19:7420-7427. 